\title{
On the universality in the extensional rheology of monodisperse polymer melts and oligomer dilutions thereof
}

\author{
Rasmussen, Henrik; Wingstrand, Sara Lindeblad; Hassager, Ole
}

\section{Published in:}

Rheologica Acta

Link to article, DOI:

10.1007/s00397-019-01156-w

Publication date:

2019

Document Version

Peer reviewed version

Link back to DTU Orbit

Citation (APA):

Rasmussen, H., Wingstrand, S. L., \& Hassager, O. (2019). On the universality in the extensional rheology of monodisperse polymer melts and oligomer dilutions thereof. Rheologica Acta, 58(6-7), 333-340.

https://doi.org/10.1007/s00397-019-01156-w

\section{General rights}

Copyright and moral rights for the publications made accessible in the public portal are retained by the authors and/or other copyright owners and it is a condition of accessing publications that users recognise and abide by the legal requirements associated with these rights.

- Users may download and print one copy of any publication from the public portal for the purpose of private study or research.

- You may not further distribute the material or use it for any profit-making activity or commercial gain

- You may freely distribute the URL identifying the publication in the public portal 


\title{
On the universality in the extensional rheology of monodisperse polymer melts and oligomer dilutions thereof
}

\author{
Henrik Koblitz Rasmussen ${ }^{1}$, Sara Lindeblad Wingstrand ${ }^{2}$ and Ole Hassager ${ }^{2}$ \\ (1) Department of Mechanical Engineering \\ (2) Department of Chemical and Biochemical Engineering, \\ Technical University of Denmark, DK-2800 Kgs. Lyngby, Denmark
}

\begin{abstract}
The startup and steady extensional viscosities were measured on two narrow molar mass distributed (NMMD) poly-methyl-methacrylates (PMMA) diluted in 57\% $2.1 \mathrm{~kg} /$ mole oligomer MMA. The oligomer is short enough to be random configured and un-entangled though it is still a Kuhn chain. The weight based average molecular weights of the PMMAs are $86 \mathrm{~kg} / \mathrm{mole}$ and $270 \mathrm{~kg} /$ mole with polydispersites of 1.08 and 1.09 respectively. The extensional viscosities were in theoretical agreement with a constant 'interchain pressure' model, representing the maximal level of strain hardening in a Kuhn fluid. This has been observed for similar (styrene) oligomer diluted NMMD polystyrene before. The original 'interchain pressure' model by Marrucci and Ianniruberto (2004) (Macromolecules 37(10):3934-3942), represents the minimal level of strain hardening in a Kuhn fluid. It has been shown previously to predict the extensional viscosities of NMMD polystyrene melts and it is also in agreement with the extensional viscosities of the $86 \mathrm{~kg} /$ mole NMMD PMMA melt as well.
\end{abstract}

\section{Introduction}

The physics of polymer melt flow is still not established. Of particular interest is its use in modelling of plastic flow in a production process. In this case constitutive equations capable of continuum mechanical simulations of the complex flow of polymer melts correctly are needed. Experimentally, to generate experiment with information of the flow physics of polymer melts, efforts have been put into flow measurement on theoretical ideal monodisperse polymer systems. Particular uniaxial extension has been of interest due to its sensitivity to change in the material's property and composition. It was initiated by Vinogradov and co-workers (Vinogradov et al. 1975) [1], measuring the startup of uniaxial extensional stress of narrow molar mass distributed (NMMD) polyisoprenes. The samples failed at Hencky strain values at about and below 2 limiting its theoretical impact. In an effort to understand monodisperse polymer system Bhattacharjee et al. (2002) [2] measured the steady extensional viscosities on entangled MNND polystyrene solutions. The year after Bach et al. (2003b) [3] likewise measured it on MNND polystyrene melts, showing substantially less strain hardening than the solutions. Further, Nielsen et al. (2009) [4] observed that the startup of (uniaxial) extensional stresses on NMMD polyisoprene showed stress values inbetween polystyrene melts and solutions. A similar behaviour has been observed for a NMMD poly(n-butyl acrylate) (Sridhar et al. 2014) [5]. Particular to understand the difference between melt and solution behaviour Huang et al. (2013a) [6] measured the extensional viscosities of polystyrene diluted in styrene oligomer. It showed the same intermediate extensional stresses as in NMMD polyisoprene and poly(n-butyl acrylate), as long as the oligomer is a chain in term of Kuhn segments. If not, the dilutions behaved as a solvent based 
system. Entangled polymer solutions seem not to be directly relevant for polymer melts systems, whereas polymers diluted in oligomers, as long as the oligomer act as a random configured Kuhn chain appears to do so. Currently it seems as NMMD polystyrene melts behaves somewhat uniquely in extensional flow as the same lack of strain hardening has not been observed in other types of polymers melts, solutions or diluted systems. Notice that the original extensional measurements on NMMD polystyrenes by Bach et al. 2003b [3] using a filament stretching rheometer (Sridhar et al. 1991; Bach et al. 2003a) [7, 8] have been reproduced by independent researchers using (Luap et al. 2005) [9] a Meissner Rheometer (Meissner and Hostettler 1994) [10].

Here we will study the extensional viscosities in MNND poly methyl methacrylate (PMMA), both as pure melt and oligomer dilutions. New extensional series on NMMD PMMA oligomer dilutions will be presented, measured in a wider range of extensional rates compared to the ones measured by Wingstrand et al. (2015) [11].

\section{Materials and characterization}

\subsection{Materials}

We particular investigate the extensional viscosity of two narrow molar mass distributed (NMMD) poly methyl methacrylates (PMMA) oligomer dilutions at the same concentration. It involves a ( $43 \%$ weight fraction) $86 \mathrm{~kg} /$ mole and a $270 \mathrm{~kg} /$ mole PMMA diluted in $2.1 \mathrm{~kg} / \mathrm{mole}$ oligomer methyl methacrylates (OMMA). The polydispersites $M_{w} / M_{n}$ were measured to 1.08 and 1.09 for the 86 $\mathrm{kg} /$ mole and a $270 \mathrm{~kg} /$ mole PMMA polymers, respectively. The polydispersity of the OMMA was 1.22. The average molecular weights are weight based, i.e. $M_{w} . M_{n}$ is the mole based average molecular weight. The PMMA polymers are the same materials as in Wingstrand et al. (2015) [11] and the molecular weights were measured by Wingstrand et al. (2015) [11] as well (listed in table 1), using size exclusion chromatography (SEC). The weight fraction of the PMMA in the oligomer dilutions, $\theta$ (table 2), were confirmed by the peak areas of the bimodal curve in the SEC. The Glass transition temperatures, $T_{g}$, were measured by differential scanning calorimetry (DSC). About $4 \mathrm{mg}$ of the sample was positioned in a hermeneutic pan using a TA Q1000 with a heating of $10^{\circ} \mathrm{C} /$ minute. All materials were prepared and samples shaped using the procedures described in Wingstrand et al. (2015) [11].

\subsection{Mechanical spectroscopy}

The PMMA270k/OMMA2k blend (see table 2) and PMMA86k melt (see table 2) were characterized with mechanical spectroscopy by Wingstrand et al. (2015) [11]. The mechanical spectroscopy of the samples, under $N_{2}$ atmosphere, were performed using an Ares G2 rheometer from TA Instruments. The small amplitude oscillatory shear measurements were using an $8 \mathrm{~mm}$ plate-plate geometry with a gap of about $0.8 \mathrm{~mm}$. All measurements where time temperature shifted to a temperature of $150^{\circ} \mathrm{C}$. The same procedure was applied to the PMMA86k/OMMA2k blend (see table 2) here.

To quantify the small amplitude oscillatory shear measurements by linear viscoelasticity the memory function, $M\left(t-t^{\prime}\right)$, from Baumgaertel, Schausberg and Winter (BSW) (Baumgaertel et al. 1990, Baumgaertel and Winter 1992) $[12,13]$ is used here. It was given as

$$
\begin{aligned}
M\left(t-t^{\prime}\right) & =\int_{0}^{\infty} \frac{H(\tau)}{\tau^{2}} e^{\left(-\left(t-t^{\prime}\right) / \tau\right)} d \tau, \\
H(\tau) & =n_{e} G_{N}^{0}\left[\left(\frac{\tau}{\tau_{d}}\right)^{n_{e}}+\left(\frac{\tau}{\tau_{c}}\right)^{-n_{g}}\right] h\left(1-\tau / \tau_{d}\right) .
\end{aligned}
$$


$h(x)$ is the Heaviside step function where $t$ and $t^{\prime}$ are the present and past time respectively. $\tau_{d}$ is the maximal relaxation time and $\tau_{c}$ is the characteristic relaxation time of the glassy modes. $G_{N}^{0}$ is the plateau modulus. As in Rasmussen and Huang (2017) [14] we use the fixed values of $n_{e}=0.2$ and $n_{g}=0.7$ for all PMMA samples. The remaining three linear viscoelastic parameters are obtained by fitting the measured mechanical spectroscopic data to the BSW model (Rasmussen et al. 2000) [15]. The parameters for the PMMA270k/OMMA2k blend were obtained by Rasmussen and Huang (2017) [14]. The mechanical spectroscopic measurements for the PMMA86k melt (from Wingstrand et al. 2015) [11] and the PMMA86k/OMMA2k blend with the corresponding fittings are shown in figure 1 .

\subsection{Extensional Measurements}

The extension measurements on the PMMA blends were performed using an in-house developed filament stretching rheometer (FSR) (Bach at al 2003, Marin et al 2013) [15] [16]. The PMMA blends were moulded into cylindrical shaped samples with initial diameter, $D_{i}$, of $5.4 \mathrm{~mm}$ and heights, $L_{i}$, of 1.5-2.5 mm. $D(t)$ is the central diameter. The samples were pre-stretched, with a rate considerably slower than the inverse largest relaxation time of the particular sample, to a central diameter, $D_{0}$, in the range of 2.5-5.2 mm. The Hencky strain is calculated as $\epsilon(t)=-2 \ln \left(D(t) / D_{0}\right)$ and the extensional viscosity as $\eta_{E}^{+}=\left(\sigma_{\mathrm{zz}}-\sigma_{\mathrm{rr}}\right) / \dot{\epsilon}_{0} . \sigma_{\mathrm{zz}}$ and $\sigma_{\mathrm{rr}}$ are the axial (the drawing direction) and radial components of the stress tensor, $\boldsymbol{\sigma}$, respectively. $\dot{\epsilon}_{0}$ is the (constant) applied extensional rate, defined as $\dot{\epsilon}=d \epsilon / d t$. The sample is stress relaxed before the initiation of the stretch (at $t=0$ ). At small values of the Hencky strain, pre-strain and aspect ratio a shear contribution may add to the measured extensional viscosity. We have used the stress correction formula from Rasmussen et al. (2010) [17] to obtain the correct, ideally within 3\%, initial extensional viscosities in these cases. All the extension measurements were time-temperature shifted to $150^{\circ} \mathrm{C}$.

\section{Constitutive models}

A variety of models considering the flow of polymer systems have been published in the recent years, meant to predict the fluid mechanics of polymer systems in general (Yaoita et al. 2012, Park et al. 2012, Andreev et al. 2013) [18, 19, 20]. Concerning monodisperse polymer melts system Doi and Edwards $(1978 ; 1986)$ [21] initiated the constitutive modelling with a model based on pure orientational stress. The idea of chain stretch was introduced by Marrucci and co-workers (Marrucci and Cindio 1980, Marrucci and Grizzuti 1988) [22, 23]. All the following suggested constitutive equations were based on a chain stretch. It is generally accepted and shown in both shear (Osaki et al. 1982; Menezes and Graessley 1982; Takahashi et al. 1993) [24, 25, 26] and extension (Bhattacharjee et al. 2002) [2] that the strain hardening in the flow depends on the Rouse time, $\tau_{R}$, all suggesting a dependence of the Rouse time as the square of the number of entanglements, $Z$. Particular concerning monodisperse systems, to explain the transition from melts to solutions, the interchain pressure (Wagner 2014) [27] and monomeric friction (Yaoita et al. 2012; Ianniruberto at al 2012) $[18,28]$ have been considered. None of the referred models, and any other according to our knowledge, seems to be able to address the differences in NMMD melt uniaxial extensional behaviour.

Here we will analyse the extensional viscosities using on the molecular stress function (MSF) constitutive framework (Wagner et al. 2005; 2010) [29, 30]. In the MSF constitutive framework the components, $\sigma_{\mathrm{ij}}$, of the stress tensor were defined as

$$
\sigma_{\mathrm{ij}}=\int_{-\infty}^{t} M\left(t-t^{\prime}\right) f\left(\boldsymbol{x}, t, t^{\prime}\right)^{2} 5\left\langle\frac{\left[\mathbf{E}\left(\boldsymbol{x}, t, t^{\prime}\right) \cdot \mathbf{u}\right]\left[\mathbf{E}\left(\boldsymbol{x}, t, t^{\prime}\right) \cdot \mathbf{u}\right]}{\left|\mathbf{E}\left(\boldsymbol{x}, t, t^{\prime}\right) \cdot \mathbf{u}\right|^{2}}\right\rangle d t^{\prime}
$$


with an initial value of the molecular stress function of $f\left(\boldsymbol{x}, t^{\prime}, t^{\prime}\right)=1$. Here $\mathbf{E}\left(\boldsymbol{x}, t, t^{\prime}\right)$ is the displacement gradient tensor. Its components are defined as $E_{\mathrm{ij}}\left(\boldsymbol{x}, t, t^{\prime}\right)=\partial x_{\mathrm{i}} / \partial x_{\mathrm{j}}{ }^{\prime}, \mathrm{i}=1,2,3$ and $\mathrm{j}=1,2,3 . \boldsymbol{x}=\left(x_{1}, x_{2}, x_{3}\right)$ and $\left(x_{1}^{\prime}, x_{2}^{\prime}, x_{3}^{\prime}\right)$ are the Cartesian coordinates of the same particle in the present, $t$, and past time, $t^{\prime}$, respectively. All angular brackets are unit sphere integral, given as $\langle\ldots\rangle=1 /(4 \pi) \int_{|\mathbf{u}|=1} \ldots d \mathbf{u}$. $\mathbf{u}$ is the unit vector. The analytical solution of all involved unit sphere integrals can be found in Urakawa et al. (1995) [31].

Here we will particularly consider two constitutive models. Both based on the interchain pressure (Marrucci and Ianniruberto 2004) [33]. Marrucci and Ianniruberto (2004) [33] suggested that in a monodisperse polymer melt the interchain pressure increases as the chains extend. This effectively reduces the strain hardening to a minimum level. Rasmussen and Huang (2014) [34] suggested that in an oligomer dilution the oligomer Kuhn chain will be in a random configuration imposing a constant interchain pressure on the long polymers. This maximized the strain hardening in a structured (i.e. Kuhn) fluid. In a MSF framework (Wagner et al. 2005) [29] the interchain pressure stretch evolution is given as

$$
\frac{\partial}{\partial t} f\left(\boldsymbol{x}, t, t^{\prime}\right)=f\left(\boldsymbol{x}, t, t^{\prime}\right)\left[\frac{\partial}{\partial t}\left\langle\ln \left|\mathbf{E}\left(\boldsymbol{x}, t, t^{\prime}\right) \cdot \mathbf{u}\right|\right\rangle-\frac{f\left(\boldsymbol{x}, t, t^{\prime}\right)}{3 \tau_{R}}\left(f\left(\boldsymbol{x}, t, t^{\prime}\right)^{3}-1\right)\right] .
$$

for the model suggested by Marrucci and Ianniruberto (2004) [33], and

$$
\frac{\partial}{\partial t} f\left(\boldsymbol{x}, t, t^{\prime}\right)=f\left(\boldsymbol{x}, t, t^{\prime}\right)\left[\frac{\partial}{\partial t}\left\langle\ln \left|\mathbf{E}\left(\boldsymbol{x}, t, t^{\prime}\right) \cdot \mathbf{u}\right|\right\rangle-\frac{1}{\tau_{R}}\left(c\left(f\left(t, t^{\prime}\right)\right) f\left(\boldsymbol{x}, t, t^{\prime}\right)-1\right)\right]
$$

in the case of the constant 'interchain pressure' (Rasmussen and Huang 2014) [34]. These depend on the Rouse time, $\tau_{R}$. In the latter one Rasmussen and Huang (2014) [34] used the transition to a maximum extensibility as the relative Padé (Cohen 1991) [35] inverse Langevin function as suggested by Ye and Sridhar (2005) [36]:

$$
c(f)=\frac{\left(3-f^{2} / \lambda_{\max }^{2}\right)\left(1-1 / \lambda_{\max }^{2}\right)}{\left(3-1 / \lambda_{\max }^{2}\right)\left(1-f^{2} / \lambda_{\max }^{2}\right)} .
$$

$\lambda_{\max }$ is the maximal relative stretch. The stretch is the square root of the number of Kuhn steps between entanglements for the particular polymer, given as

$$
\lambda_{\max }=\sqrt{N_{e}}=\sqrt{N_{K} / Z} .
$$

$N_{K}=M_{w} / M_{K}$ is the number of Kuhn steps in the polymer chain where $M_{K}$ is the Kuhn step molecular weight. It is expected to be independent of the degree of the oligomer dilution, $\theta$. As in Rasmussen and Huang (2017) [14] we will use the $M_{K}$ value of atactic PMMA of $0.6 \mathrm{~kg} / \mathrm{mole}$ (Fetters et al. 2007) [37]. Both PMMAs were synthesized (Wingstrand et al. 2015) [11] by living anionic polymerization using the same initiator giving the same tacticity, although the stereoisomeric structures of involved PMMAs are not clearly defined. A linear dependency on the number of entanglements with the concentration, $Z=M_{w}\left(\theta / M_{e}\right)$, is expected. As in Rasmussen and Huang (2014) [34] we use the value of the entanglement molecular weight $M_{e}$ of $5.4 \mathrm{~kg} / \mathrm{mole}$ for both involved PMMA melts. The value of $5.4 \mathrm{~kg} /$ mole for $M_{e}$ lies between the 5 to $6 \mathrm{~kg} / \mathrm{mole}$ given by Wingstrand et al. (2015) [11]. This interval was based on the assumption of identical mechanical spectroscopic data with the same number of entanglements. A comparison with MNND polystyrenes was made, with the assumption of an entanglement molecular weight of $13.3 \mathrm{~kg} /$ mole for polystyrene.

\section{Results and Discussion}

The Rouse time, scaled relative to the relaxation time, depends on the number of entanglement. In Rasmussen and Huang (2017) [14] the Rouse time was fitted to a set of extensional viscosities 
measured using the same 270k NMMD PMMA as the one here though diluted in $56 \% 3.5 \mathrm{~kg} / \mathrm{mole}$ OMMA. With an expected squared dependency of the Rouse time on the entanglement number a relation of

$$
\tau_{d} / \tau_{R}=50 \cdot(Z / 22)^{2}
$$

were obtained. We use the same relation her. Notice the proportionality factor (here of 50 for PMMA) is not identical to the corresponding values for other polymers (Rasmussen 2015) [38].

In figure 2 and 3 the extensional measurements on the two oligomer dilutions are presented, showing the extensional viscosities as a function of the time at increasing extensional rates from the right to the left curves. The Rouse times, $\tau_{R}$, have been marked on the figures by the arrows on the time axis. This represents the shift between an extensional flow dominated by pure configurational orientations at large time scales, and the strain hardening occurring at fast flows. We have added the corresponding theoretical model predictions to the experiments for the constant interchain pressure with (i.e. $\lambda_{\max }^{2}=20.9$ ) and without (i.e. an infinite value of $\lambda_{\max }$ ) the maximal extensibility, applying the equations (5) and (6). These are the solid and dashed lines in the figures respectively. Due to the different polymer lengths in the two samples the Rouse time, relative to the relaxation time, is a factor of 10 different to each other. A good agreement between the models based on the constant interchain pressure assumption has been obtained in the startup of the extensional flow region, confirming both the use of the relation for the Rouse time and the constant interchain pressure, as a model for these diluted systems. With the use of the same oligomer concentration of $57 \%$ in the two OMMA dilution used here, they ideally have the same maximal extensibility with a $\lambda_{\max }$ value of about 4.6. With the use of the limit imposed by the chain extensibility the transition to extensional viscosity shows a maximal deviation of about $20 \%$ compared to the experiments. We have also added the model prediction from the equation (4), ideally expected to be a model for the flow behaviour of an undiluted monodisperse polymer melt, as suggested by Marrucci and Ianniruberto (2004) [33]. It is represented by equation (4). It is the dashed dotted line in the figures 2 and 3. The model predictions stay as expected well below the experimental values. Equation (4) had been presented without a maximal extensibility. The inclusion of a maximal extensibility in this equation has currently not been relevant. At the extensional conditions presented in figure 2 and 3 steady levels of the viscosities will be lowered, somewhat, further below the experimental values if a maximal extensibility is included. Though it is not theoretically clear exactly how it should formulated.

The two models quantified in equation (4) and (5) represent the minimal and maximal level of strain hardening in a Kuhn fluid, respectively. NMMD polystyrene melts follows the minimal level (equation (4)). Intermediate behaviour has actually been observed in the extension of NMMD polystyrene dilutions with styrene oligomer concentration of $10 \%$ and $30 \%$ (Huang and Rasmussen 2017) [39]. At higher oligomer concentrations, shown at and above 47\%, the assumption of the constant interchain pressure assumption (equation (5)) seems to hold accurately. The involved styrene oligomers were all Kuhn chains without entanglements. The relation in (5) has been shown to be valid for a wide range of oligomer MNND polystyrene dilutions (Huang and Rasmussen 2017) [39] as well on bidisperse systems of polystyrene and polyisoprene [38], as long as the short chains are in random configurations. More surprisingly agreement with the constant interchain pressure in equation (5) has been observed for NMMD polyisoprene (Rasmussen 2016) [40] and poly(n-butyl acrylate) melts as well (Sridhar et al. 2014) [5].

The use of the maximal extensibility seems to be valid for the diluted NMMD PMMA systems. Previously the validity of the maximal extensibility has currently only been confirmed in bidisperse, including oligomer, polystyrene systems. Experimentally it is a challenging task to measure the steady extensional viscosities, $\eta_{E}$ (see figure 4 ). The maximal extensibility has not been included in equation (4), though the maximal extensibility is theoretically at its lowest value in monodisperse melts. Currently in NMMD polystyrene melts the measured extension rates have not been sufficiently 
high to create a significant effect of the inclusion of a maximal extensibility in equation (4).

In figure 5 we have shown the measured startup of extensional viscosities by Alvarez et al. (2016) on the undiluted 86k NMMD PMMA melt, measured at four different extensional rates. Alvarez et al. (2016) measured the startup of extensional viscosities on the same PMMA batch as the one here, and used the same FSR as the one used here. The 86k NMMD PMMA has a number of entanglement of 15.9. This is inbetween the corresponding values for the two dilutions as seen in table 2, having 6.8 and 21.5 entanglements, respectively. The arrow on the time axis in figure 5 is the Rouse time of the melt. Both interchain models quantified in equation (4) and (5) have been added in the figure as well. The latter one with a maximal extensibility $\lambda_{\max }$ of 3 , the ideal value for the undiluted PMMA. This is a value somewhat lower than the value of 4.7 in polystyrene. As in MNND polystyrene melts the agreement seems to be in accordance with the idea of Marrucci and Ianniruberto (2004) [33]. Further, the measurements cannot be used to conclude on an effect of a potential maximal extensibility in the use of equation (4).

\section{Summary and Conclusion}

We have measured the startup of uniaxial extensional viscosities until the steady viscosity on two oligomer diluted NMMD poly(methyl methacrylate)s, both diluted in $57 \% 2.1 \mathrm{~kg} / \mathrm{mole}$ oligomer methyl methacrylate. Both dilutions, having 6.8 and 21.5 entanglements, were measured within broad ranges of extensional rates, below and above the Rouse time. Both extensional viscosity series follow a MSF constitutive model, based on a constant interchain pressure. It is a model for the flow of an idealized solution of a monodisperse polymer in a structured diluent (i.e. consisting of Kuhn chains), appearing in a random configuration. This is the maximal possible level of extensional strain hardening in an ideally structured fluid. The steady viscosities were in agreement with a finite chain extensibility of (undiluted) PMMA of 3, based on the Kuhn steps in one entanglement. Further the uniaxial extensional viscosities measured by Alvarez et al. (2016) on an undiluted NMMD poly(methyl methacrylate) melt, with 15.9 entanglement, follows the interchain pressure model by Marrucci and Ianniruberto (2004) [33] representing the minimal hardening in a Kuhn fluid. MNND PMMA melts and (oligomer methyl methacrylate) dilutions behaved theoretically similar to NMMD polystyrenes systems. 
Table 1: NMMD poly methyl methacrylates (PMMA) and oligomer methyl methacrylates (OMMA) molecular weights from Wingstrand et al. (2015) [11].

\begin{tabular}{llr}
\hline Name & $M_{w}$ & $M_{w} / M_{n}$ \\
\hline OMMA2k & $2.1 \mathrm{~kg} /$ mole & 1.22 \\
PMMA86k & $86 \mathrm{~kg} /$ mole & 1.08 \\
PMMA270k & $270 \mathrm{~kg} /$ mole & 1.09 \\
\hline
\end{tabular}

Table 2: Linear viscoelastic parameters of the poly methyl methacrylates (PMMA) melt and blends at $150^{\circ} \mathrm{C}$. The molecular weights are listed in table 1 . The weight fraction of the largest polymer chains in the polymer/oligomer blend is $\theta$. The values of $n_{e}=0.2$ and $n_{g}=0.7$. The remaining relevant parameters are obtained from mechanical spectroscopical measurements, as shown in figure 1, fitting the parameters of the BSW model (2) (Rasmussen et al. (2000)). The parameters for the PMMA270k/OMMA2k blend were obtained by Rasmussen and Huang (2017) [14]. The values $M_{k}=0.6 \mathrm{~kg} /$ mole and $M_{e}=5.4 \mathrm{~kg} /$ mole have been used in the calculation of the number of Kuhn steps, $N_{K}$, and the number of entanglements, $Z$, respectively.

\begin{tabular}{l|rrrrrrr}
\hline Melts & $\theta$ & $G_{N}^{0}$ & $\tau_{d}$ & $\tau_{c}$ & $Z$ & $N_{K} / Z$ & $T_{g}$ \\
\hline PMMA86k & 1 & $1150 \mathrm{kPa}$ & $550 \mathrm{~s}$ & $0.15 \mathrm{~s}$ & 15.9 & 9 & $122.8^{\circ} \mathrm{C}$ \\
PMMA86k/OMMA2k & 0.43 & $130 \mathrm{kPa}$ & $5.6 \mathrm{~s}$ & $0.02 \mathrm{~s}$ & 6.8 & 20.9 & $90.4^{\circ} \mathrm{C}$ \\
PMMA270k/OMMA2k & 0.43 & $130 \mathrm{kPa}$ & $1000 \mathrm{~s}$ & $0.04 \mathrm{~s}$ & 21.5 & 20.9 & $108.6^{\circ} \mathrm{C}$ \\
\hline
\end{tabular}




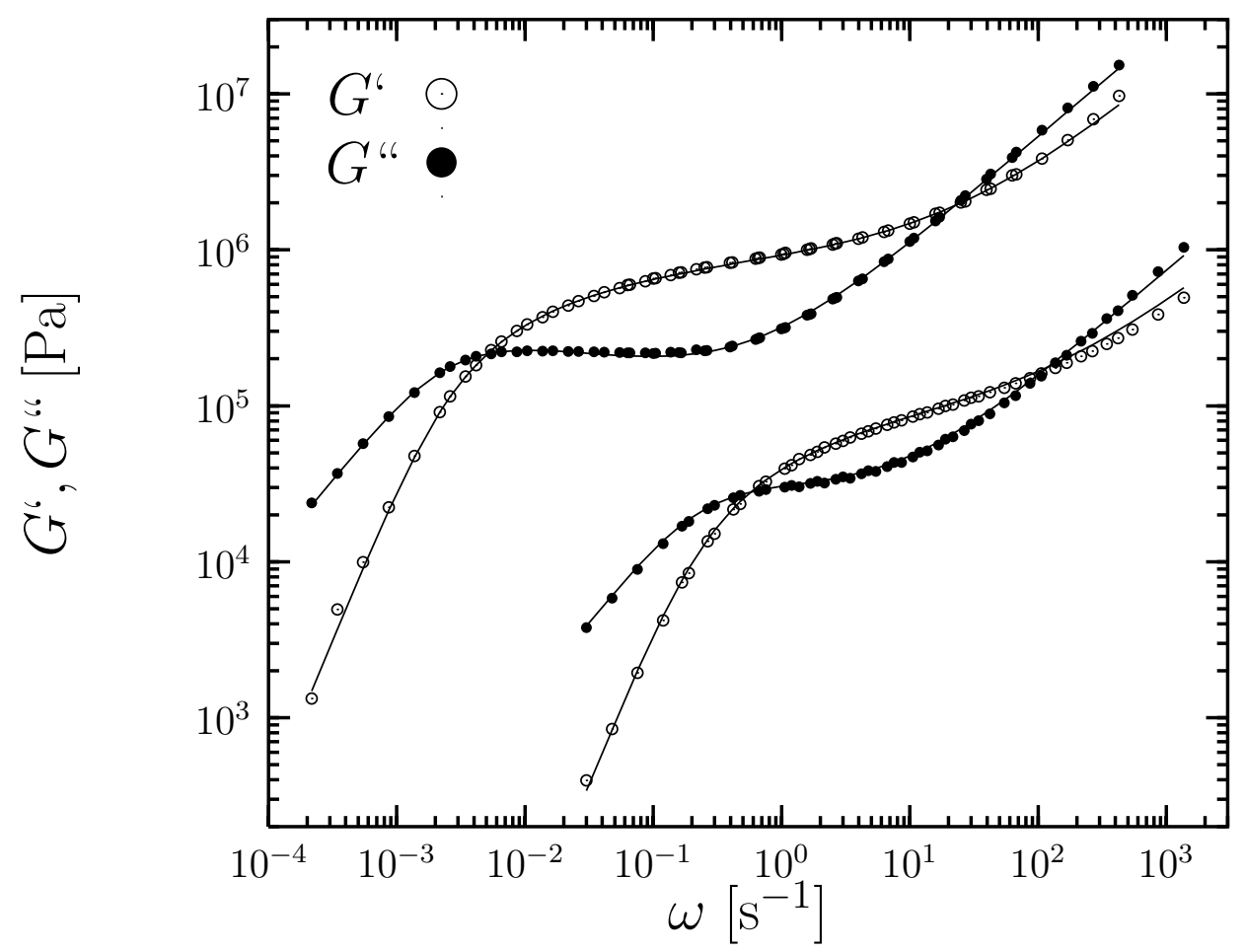

Figure 1: Loss, $G^{\prime \prime}$ (open circles; ○) and storage moduli, $G^{\prime}$ (bullets; •), both as a function of the angular frequency $\omega$ measured at $150^{\circ} \mathrm{C}$ for the $\theta=0.43(43 \%)$ PMMA86k/OMMA2k blend and PMMA86k melt. The solid lines (- ${ }^{-}$) are the least-square fittings to the BSW model in equation $(2)$. 


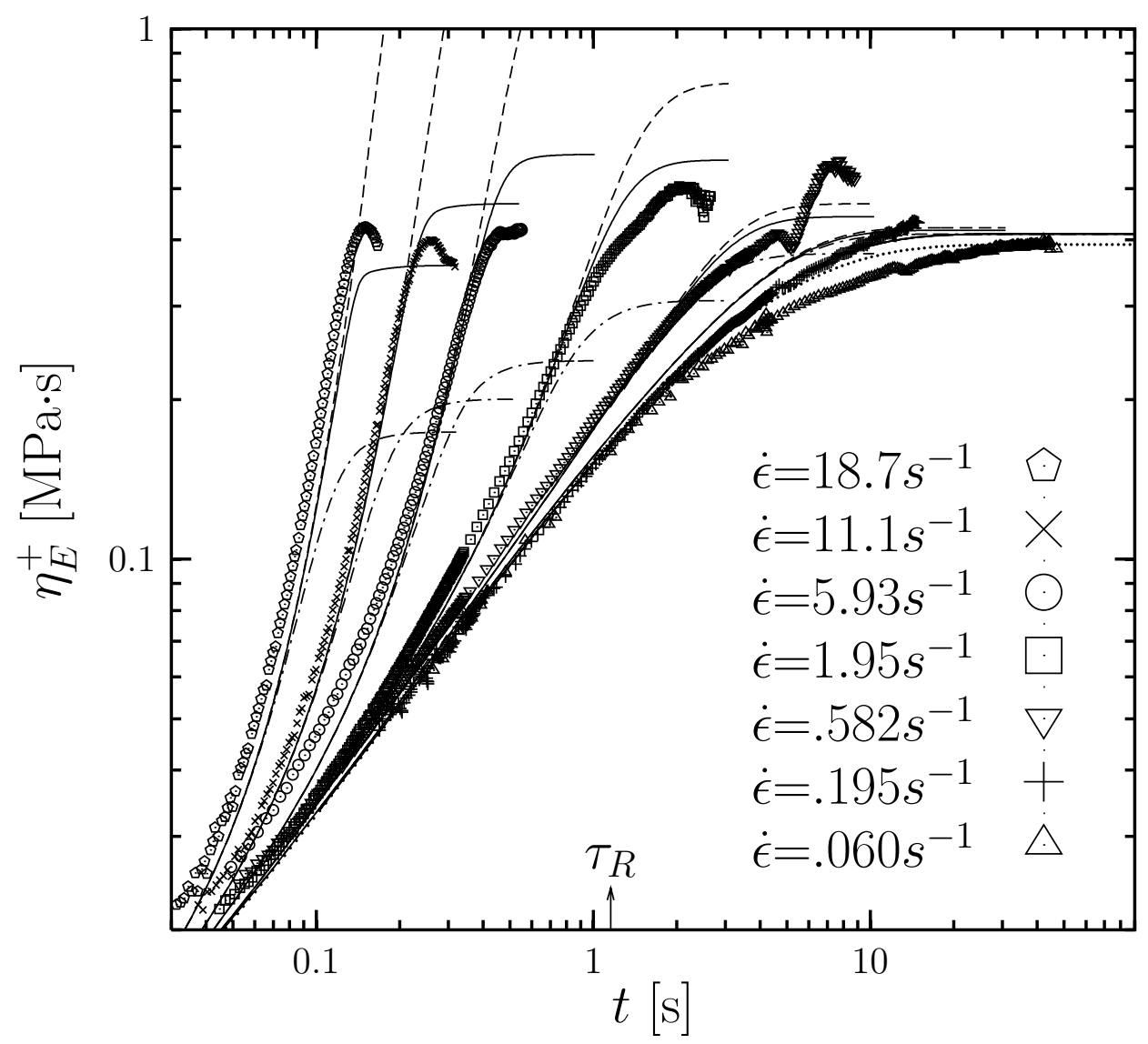

Figure 2: The startup of extensional viscosity, $\eta_{E}^{+}$, as a function of time at $150^{\circ} \mathrm{C}$. The symbols are measurements on the $(\theta=0.43)$ PMMA86k/OMMA2k blend $(43 \% 86 \mathrm{~kg} /$ mole diluted in the 2.1 $\mathrm{kg} /$ mole oligomer $)$. The dots $(\cdots)$ are the linear viscoelastic extensional viscosity using equation $(2)$ with the parameters listed in table 2 . The solid lines $(-)$ are the constant interchain pressure model equation (5) with a maximum extensibility of $\lambda_{\max }^{2}=20.9$. The dashed lines (- - -) are the corresponding predictions to the data from the equation (5) without a limit on the chain extensibility. The dashed-dotted lines (- - - ) are the corresponding predictions to the data from the equation $(4)$. 


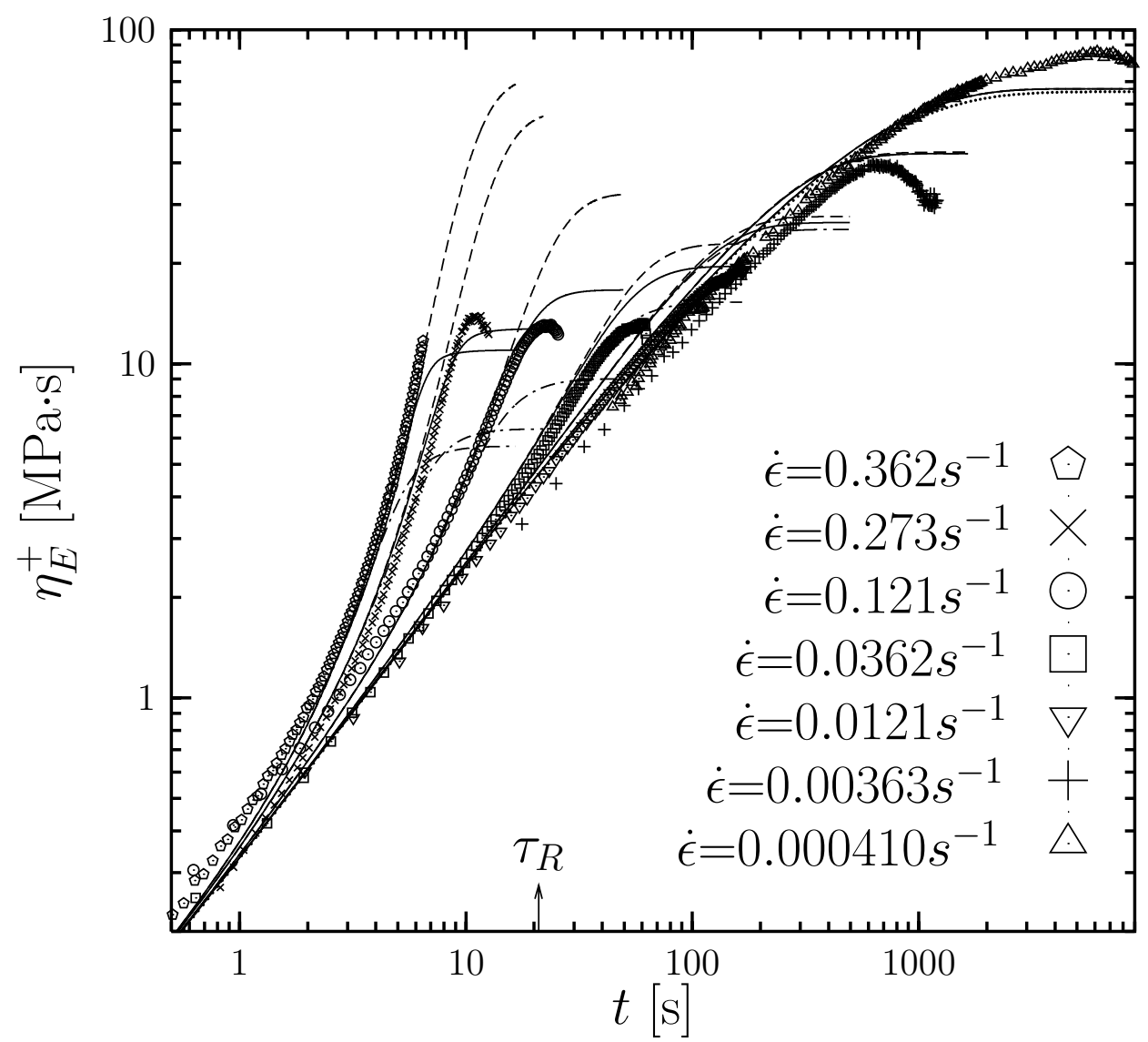

Figure 3: The startup of extensional viscosity, $\eta_{E}^{+}$, as a function of time at $150^{\circ} \mathrm{C}$. The symbols are measurements on the $(\theta=0.43)$ PMMA270k/OMMA2k blend $(43 \% 270 \mathrm{~kg} / \mathrm{mole}$ diluted in the $2.1 \mathrm{~kg} /$ mole oligomer $)$. The dots $(\cdots)$ are the linear viscoelastic extensional viscosity using equation (2) with the parameters listed in table 2 . The solid lines $(-)$ are the constant interchain pressure model equation (5) with a maximum extensibility of $\lambda_{\max }^{2}=20.9$. The dashed lines (- - -) are the corresponding predictions to the data from the equation (5) without a limit on the chain extensibility. The dashed-dotted lines (- - - -) are the corresponding predictions to the data from the equation (4). 


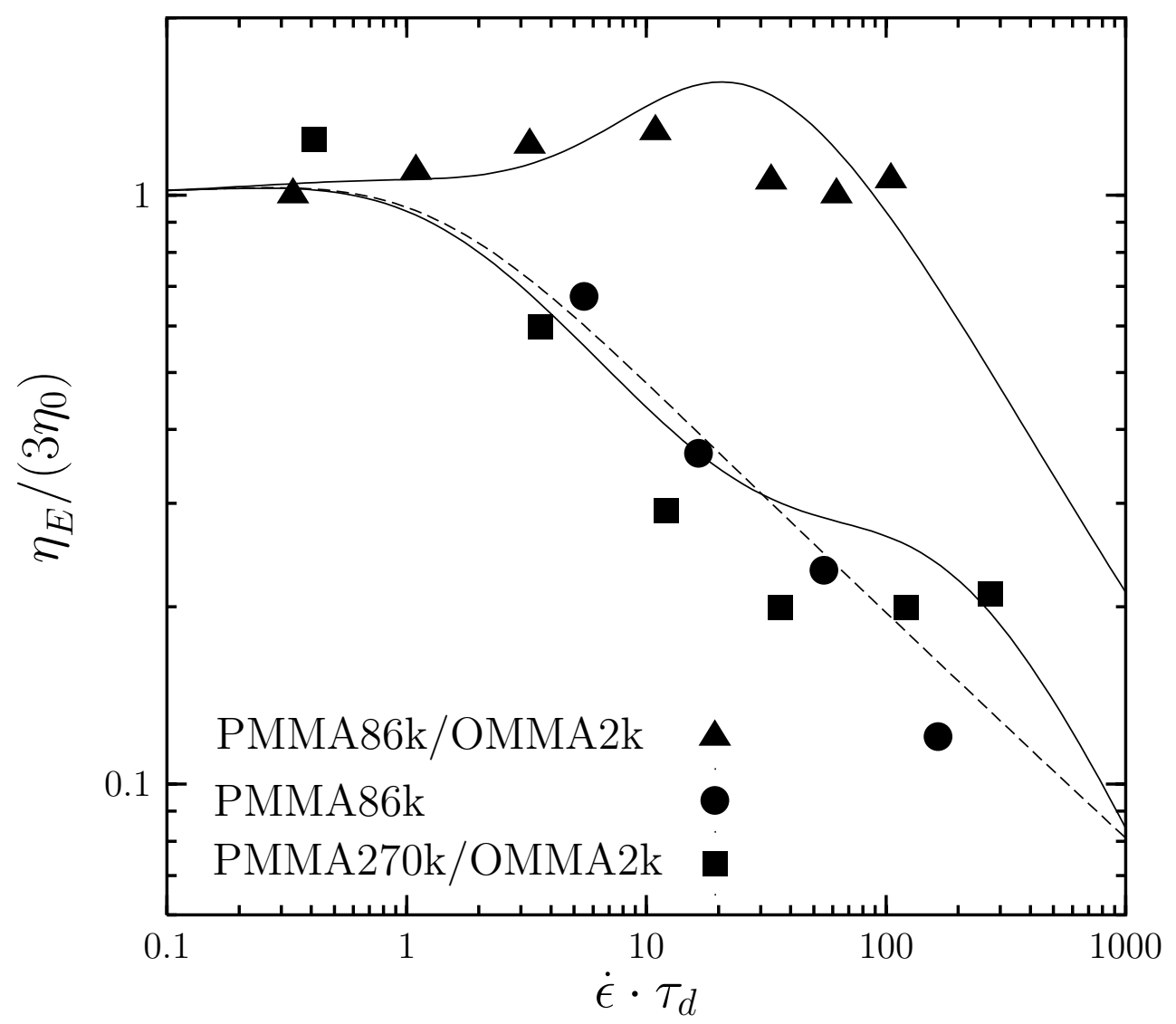

Figure 4: Steady extensional viscosity, $\eta_{E}$, relative to the zero extensional rate viscositiy, $3 \eta_{0}$, as a function of the extensional rate, $\dot{\epsilon}$, multiplied with the maximal relaxation time, $\tau_{d}$. The solid lines (-) are the corresponding predictions to the PMMA270k/OMMA2k (triangles) and PMMA86k/OMMA2k data (boxes) using the constant interchain pressure model equation (5), both lines with a maximum extensibility of $\lambda_{\max }^{2}=20.9$. The dashed line (- - ) is the interchain pressure model from equation (4) corresponding predictions to the 86k NMMD PMMA melt data (bullets) from Alvarez et al. (2016). 


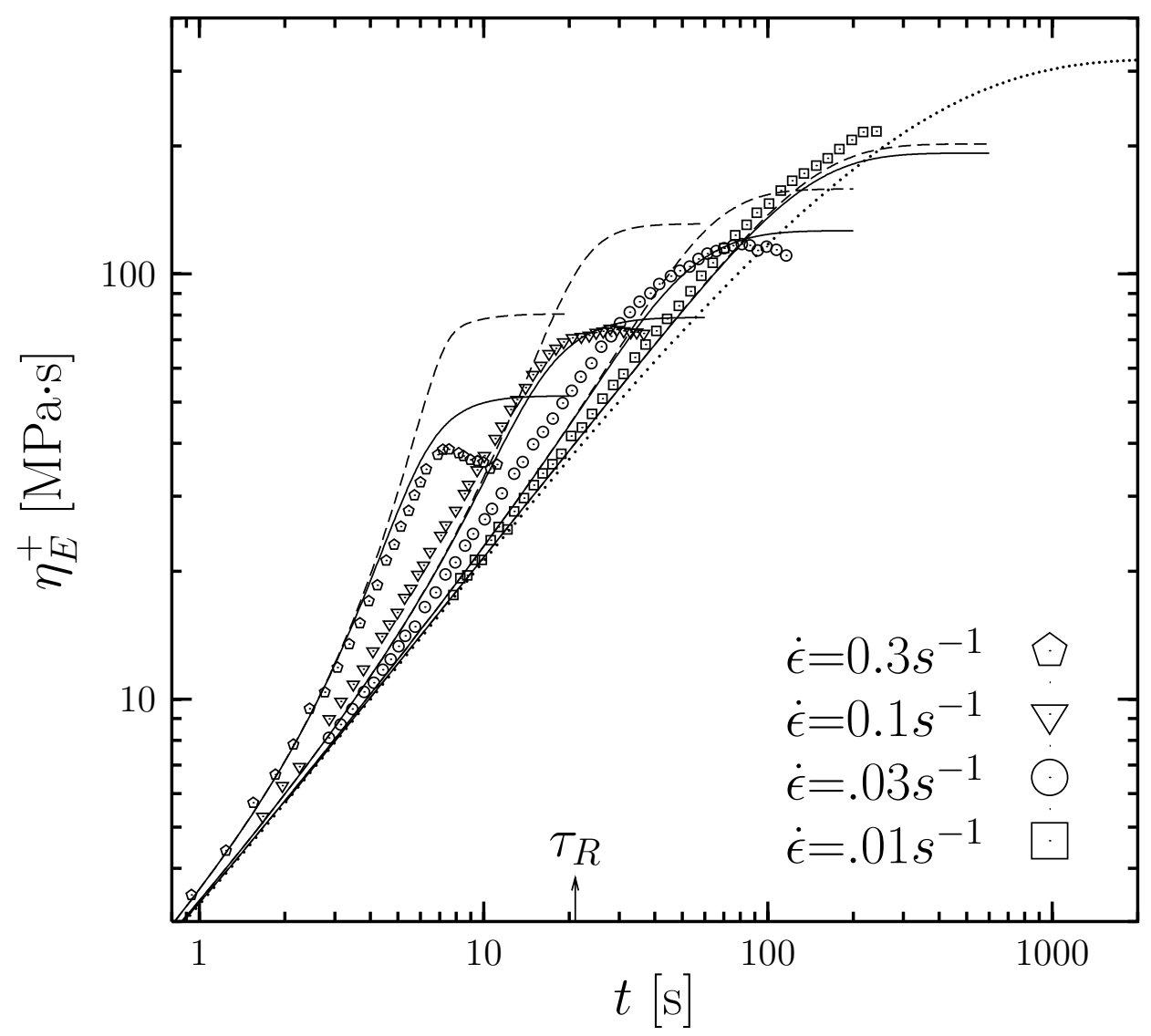

Figure 5: The startup of extensional viscosity, $\eta_{E}^{+}$, as a function of time at $150^{\circ} \mathrm{C}$. The dots $(\cdots)$ are the linear viscoelastic extensional viscosity using equation (2) with the parameters listed in table 2. The solid lines (-) are the interchain pressure model from equation (4). The dashed lines (- - ) are the corresponding predictions to the data from equation (5) with the limit on the chain extensibility of $\lambda_{\max }=3$.

\section{References}

[1] Vinogradov GV, Malkin AY, Volosevitch VV, Shatalov VP, Yudin VP (1975) Flow, high-elastic (recoverable) deformation, and rupture of uncured high molecular weight linear polymers in uniaxial extension. Journal of Polymer Science: Polymer Physics Edition 13(9):1721-1735.

[2] Bhattacharjee PK, Oberhauser JP, McKinley GH, Leal LG, Sridhar T (2002) Extensional rheometry of entangled solutions. Macromolecules 35(27):10131-10148.

[3] Bach A, Almdal K, Rasmussen HK, Hassager O (2003b) Elongational viscosity of narrow molar mass distribution polystyrene. Macromolecules 36(14):5174-5179.

[4] Nielsen JK, Hassager O, Rasmussen HK, McKinley GH (2009) Observing the chain stretch transition in a highly entangled polyisoprene melt using transient extensional rheometry. Journal of Rheology 53(6):1327-1346.

[5] Sridhar T, Acharya M, Nguyen DA, Bhattacharjee PK (2014) On the Extensional Rheology of Polymer Melts and Concentrated Solutions. Macromolecules, 47(1):379-386. 
[6] Huang Q, Mednova O, Rasmussen HK, Alvarez NJ, Skov AL, Almdal K, Hassager O (2013a) Concentrated Polymer Solutions are Different from Melts: Role of Entanglement Molecular Weight. Macromolecules 46(12):5026-5035.

[7] Sridhar T, Tirtaatmadja V, Nguyen DA, Gupta RK, Measurement of extensional viscosity of polymer solutions (1991) Journal of Non-Newtonian Fluid Mechanics 40(3):271-280.

[8] Bach A, Rasmussen HK, Hassager O (2003a) Extensional viscosity for polymer melts measured in the filament stretching rheometer. Journal of Rheology 47(2), 429-441.

[9] Luap C, Müller C, Schweizer T, Venerus DC (2005) Simultaneous stress and birefringence measurements during uniaxial elongation of polystyrene melts with narrow molecular weight distribution, Rheologica Acta 45(1):83-91.

[10] Meissner J, Hostettler J (1994) A new elongational rheometer for polymer melts and other highly viscoelastic liquids, Rheologica Acta 33(1):1-21.

[11] Wingstrand SL, Alvarez NJ, Huang Q, Hassager O (2015) Linear and Nonlinear universality in the rheology of polymer melts and solutions. Physical review letters 115(7):078302.

[12] Baumgaertel M, Schausberger A, Winter HH (1990) The relaxation of polymers with linear flexible chains of uniform length. Rheologica Acta 29(5):400-408.

[13] Baumgaertel M, Winter HH (1992) Interrelation between continuous and discrete relaxation time spectra, Journal of Non-Newtonian Fluid Mechanics 44(1):15-36.

[14] Rasmussen HK, Huang Q (2017) Constant interchain pressure effect in extensional flows of oligomer diluted polystyrene and poly(methyl methacrylate) melts. Rheologica Acta 56(1) 2734.

[15] Rasmussen HK, Christensen JH, Gøttsche SJ (2000) Inflation of polymer melts into elliptic and circular cylinders. Journal of Non-Newtonian Fluid Mechanics 93(2-3):245-263.

[16] Marin JMR, Huusom JK, Alvarez NJ, Huang Q, Rasmussen HK, Bach A, Skov AL, Hassager O (2013) A control scheme for filament stretching rheometers with application to polymer melts. Journal of Non-Newtonian Fluid Mechanics 194(1):14-22.

[17] Rasmussen HK, Bejenariu AG, Hassager O, Auhl D (2010) Experimental evaluation of the pure configurational stress assumption in the flow dynamics of entangled polymer melts. Journal of Rheology 54(6):1325-1336.

[18] Yaoita T, Isaki T, Masubuchi Y, Watanabe H, Ianniruberto G, Marrucci G (2012) Primitive Chain Network Simulation of Elongational Flows of Entangled Linear Chains: Stretch/Orientation-induced Reduction of Monomeric Friction. Macromolecules 45(6):27732782 .

[19] J. Park, D. W. Mead, and M.M. Denn, Stochastic simulation of entangled polymeric liquids in fast flows: Microstructure modification, J.Rheol. 56 (2012) 1057-1081.

[20] M. Andreev, R. N. Khaliullin, R. J. A. Steenbakkers and J. D. Schieber, Approximations of the discrete slip-link model and their effect on nonlinear rheology predictions, J. Rheol. 57 (2013) 535-557. 
[21] Doi M, Edwards SF (1978) Dynamics of concentrated polymer systems. III. Constitutive equation. Journal of the Chemical Society, Faraday Transactions II 74(1):1818-1832.

[22] G. Marrucci, B. de Cindio (1980) The stress relaxation of molten PMMA at large deformations and its theoretical interpretation, Rheologica Acta 19(1) 68-75.

[23] Marrucci G, Grizzuti N (1988) Fast flows of concentrated polymers - predictions of the tube model on chain stretching. Gazzetta Chimica Italiana 118(3):179-185.

[24] Osaki K, Inoue T, Isomura T, (2000) Stress Overshoot of Polymer Solutions at High Rates of Shear. Journal of Polymer Science part B - Polymer Physics 38(14):1917-1925.

[25] Menezes EV, Graessley WW (1982) Nonlinear rheological behavior of polymer systems for several shear-flow histories. Journal of Polymer Science part B - Polymer Physics 20(10):1817-1833.

[26] Takahashi M, Isaki T, Takigawa T, Masuda T (1993) Measurement of biaxial and uniaxial extensional flow behavior of polymer melts at constant strain rates. Journal of Rheology 37(5):827-846.

[27] Wagner MH (2014) Scaling relations for elongational flow of polystyrene melts and concentrated solutions of polystyrene in oligomeric styrene. Rheologica Acta 53(10-11):765-777.

[28] Ianniruberto G, Brasiello A, Marrucci G (2012) Simulations of fast shear flows of PS oligomers confirm monomeric friction reduction in fast elongational flows of monodisperse PS melts as indicated by rheooptical data. Macromolecules 45(19):8058-8066.

[29] Wagner MH, Kheirandish S, Hassager O (2005) Quantitative prediction of transient and steadystate elongational viscosity of nearly monodisperse polystyrene melts. Journal of Rheology 49(6):1317-1327.

[30] Wagner MH, Rolón-Garrido VH (2010) The interchain pressure effect in shear rheology. Rheologica Acta 49(5):459-471.

[31] Urakawa O, Takahashi M, Masuda T, Golshan Ebrahimi N (1995) Damping functions and chain relaxation in uniaxial and biaxial extensions: Comparison with the Doi-Edwards theory. Macromolecules 28(21):7196-7201.

[32] Doi M, Edwards SF (1986) The Theory of Polymer Dynamics; Clarendon Press: Oxford.

[33] Marrucci G, Ianniruberto G (2004) Interchain pressure effect in extensional flows of entangled polymer melts. Macromolecules 37(10):3934-3942.

[34] Rasmussen H, Huang Q (2014) Interchain tube pressure effect in extensional flows of oligomer diluted nearly monodisperse polystyrene melts, Rheologica Acta 53(3):199-208.

[35] Cohen A (1991) A Padé approximant to the inverse Langevin function. Rheologica Acta 30(3):270-273.

[36] Ye X, Sridhar T (2005) Effects of the polydispersity on rheological properties of entangled polystyrene solutions, Macromolecules 38(8):3442-3449.

[37] Fetters LJ, Lohse DJ, Colby RH (2007) Chain Dimensions and Entanglement Spacing. Physical Properties of Polymers Handbook 5(2):447-454.

[38] Rasmussen HK (2015) Interchain tube pressure effect in the flow dynamics of bi-disperse polymer melts. Rheologica Acta 54(1):9-18. 
[39] Huang Q, Rasmussen HK (2017) The transition between undiluted and oligomer-diluted states of nearly monodisperse polystyrenes in extensional flow. Rheologica Acta, 56(9):719-727.

[40] Rasmussen, HK (2016) A constitutive analysis of the extensional flows of nearly monodisperse polyisoprene melts, Polymer 104(1):251-257. 